\title{
Dossier of Disaster Recovery in AWS and Azure
}

\author{
Prasthutha $^{1}$, Chandrahasa Rai $^{2}$, Rashmi Naveen ${ }^{3}$ \\ Department of Information Science and Engineering, NMAM Institute of Technology, Nitte $e^{1,2,3}$
}

\begin{abstract}
Disaster recovery is a field of security planning that targets to protect an organization from the aftermath of significant adverse events. In this paper, we will do the scrutiny of Disaster Recovery methods in Amazon Web Service and Microsoft Azure. For any user the application developed must be available at all time. Application that is not available for a second may cause extensive complication for the business and also ruin the status of the company. For the continuity of any application the company need to have recovery plan. There are numerous cloud providers that offer service called as disaster recovery. Many of the companies get puzzled or bewilder in choosing the right provider that can match their system needs in case of any disaster. We will look forth in this paper that, how a disaster recovery process minimize the impact on the data of the application and in business operation. The management must determine the relevant events that can cause disasters and figure out their impact. They need to be firm with the goals clearly, decide suitable disaster recovery plans to choose the DRP that would be most favorable. The key parameters that need to take into consideration are basic cost, the amount of data transfer and the rate of data storage. To get the rate of uncertainty, we need to get to know the type of the disaster. Disaster maybe caused naturally or by some human errors. Cloud computing has moved to a new level of preparing for IT disasters by furnishing secondary environments for backing up and restoring data and failing over business applications. These disaster recovery resources are worthwhile and straight forward to set things up. Microsoft Azure and Amazon Web Services (AWS) are two of the top providers in terms of disaster recovery solutions currently.
\end{abstract}

Keywords: Disaster recovery, AWS, Azure, DRP, Cloud Computing

\section{INTRODUCTION}

The evolution of cloud computing technology has alternated the paradigm of the business world in exercising technology in its business processes massively. Disaster recovery is about preparing for and getting restored from a disaster. Any act or situation that has an adverse brunt on a company's business continuum or finances could be called as disaster. This comprise hardware or software breakdown, a network interruption, a power interruption, physical damage to a building like fire or flooding, human flaw, or some other important event. To curtail the impact of a calamity, companies devote time and resources to formulate and qualify, to train employees, and to archive and amend processes. The extent of investment for disaster recovery planning for an individual setup can vary effectively depending on the cost of a potential outage. Companies that have usual physical environments normally must match or copy their infrastructure to ensure the availability of extra capacity in the event of a disaster. The foundation needs to be procured, equipped, and maintained so that it is accessible to support the anticipated capacity requirements. During regular working, the infrastructure usually is under-utilized or over-provisioned. The company can upgrade its framework on an as-needed, pay-as-you-go basis with the help of Amazon Web Services (AWS). Get access to the same highly protected, trustworthy, and fast infrastructure that Amazon uses to run its own global network of websites. AWS also gives you the resilience to quickly change and optimize resources during a disaster recovery event, which can result in effective cost savings.

This paper illustrates best method to improve the disaster recovery processes, from basic investments to full-scale availability and fault tolerance, and shows you how you can use AWS services to minimize cost and ensure business progression during a Disaster Recovery situation. Azure storage is identical to S3 in that it yields cloud-based object storage for your crucial data. Azure provides its storage service as a primed service so maintenance and other problems are handled for you. You can retrieve your data from anywhere online via HTTP or HTTPS. Third-party services can also unify with Azure storage costs. The cut down costs from carrying out deviant process to enhance storage efficiency, such as data deduplication and compression. Cloud usually gives compressed stored data, but any cost savings from this are not often passed on to end users. Cloud computing provide high trustworthiness of service for preserving sensitive and important client data. At present, there are lot of DRaaS provider such as AWS, Google Cloud that offers disaster recovery in their service. Each one of the provider almost offered same features that confuses customers. Therefore we run several testing between providers that target on RTO and RPO values to guide customer for searching the best preference for their disaster recovery planning. RTO (Recovery Time Objective) and RPO (Recovery Point Objective) is the most demanding two metrics in disaster recovery planning. RTO value means the 
extent of service is nonexistent just before recovery and beginning the service repeatedly. In the other hand, RPO means highest amount of data that can be misplaced when restoration is favorable in time. A successful disaster recovery key consists of providing the service always online by the approval of SLA (Service Level Agreement) also meeting the goals of RPO and PTO. In the next division we deal with background facts regarding the DRP problem. In part 3 we compare disaster recovery plan supported by both azure and amazon AWS. We look at use of a cloud based site as a backup or secondary.

\section{BACKGROUND}

Disaster Recovery is an effort in getting things ready and restoring from a disaster. All that has atrocious effects on a company's business continual could be described as a disaster. This corresponds to hardware or software breakdown, a network interruption, a power blackout, environmental damage to a building like fire or flooding, human flaw, or some other important occurrence. Each of the company must have its own base framework to back up the business process especially that needs active requirement of application availability. Therefore, disaster recovery plan is not an alternative or preference but it is a must. Many benefits could be gained by applying disaster recovery plan, in-between help reduces the defect of critical functions, recover operations swiftly and successfully when system is down, satisfy firmness of organization, and also maintains organization's resources. Additionally disaster recovery plan grant organization or company to state higher availability and reliability of services than other competitor. There are three ways to frame disaster recovery, hot site, cold site, and warm site. Hot site means that companies have exact identical copy of their entire architecture system including server, data, storage, and network called secondary location that will not be damaged by any event altered the primary location. The data replicated between primary and secondary site. It could be active-active replication, where all databases are coincide with each other and cloned in two- way direction or active-passive replication, when data is copied in one way direction. At the same time, Cold site is the kind of way that just send backup file of the system. Warm site stand for typical way between cold and hot.

In disaster recovery details, the system to switch service from primary site to subordinate site when the service break down due to disaster is called failover. In failover state, all services along with application and data will be managed by secondary site. If not, when the primary site issues has been cleared up and the service is changed back to the primary site is called failback. Disaster recovery as a service is cloud based service bound to ensure system availability and recovery from disaster. DRaaS brought distinct way to back up important system together with application, data also resources and quickly recover systems after a disaster with lesser cost and complexity. The upper hand of carrying out DRaaS are availability, cost reduction, simplicity, visibility, scalability, flexibility.

\section{COMPARISION}

Cloud computing has shown a advanced way of preparing for IT disasters by furnishing secondary medium for backing up and restoring data and declining over business applications. These disaster recovery services are worthwhile and direct or open to set up. Microsoft Azure and Amazon Web Services (AWS) are two of the top providers of disaster recovery solutions. AWS has numerous number of distinctive cloud services accessible for usage, few of which are ideal for disaster recovery purposes. There is no appropriate AWS disaster recovery service, but the extent of preferences or choices that are available gives exceptional adaptability. Amazon S3 is a service for accumulating data as objects, which means each file is gathered in a repository without any file ranking or order. This service gives good durability, which explains that transactions are assured to save for all time even if the system crashes. S3 is perfect for mission-critical data, and using it as basic fundamental storage means the data is usable even if on premise systems fail. Amazon Glacier is a economical storage service which is optimal for data collection and backup. This service should consist of non-mission critical data. Amazon EBS allow you take snapshots of data volumes and store them on S3. The advantage of this service is that you can instantly push an EBS snapshot to a running EC2 instance, confirming the speedy return of enterprise app functionality after a failure. The EC2 instance manages the application workloads while the EBS snapshot provides fundamental primary storage for apps.

EC2 provides run down capacity in the cloud in the mode of virtual machines, which you can roll up in minutes. You can start an EC2 instance with machine images that have been configured earlier with operating systems and application stacks. This is an extensive service for building mission critical applications after a catastrophe. Route 53 is a DNS web service that can direct website visitors to your website through various endpoints in case your web servers fail after a calamity. Amazon VPC allows you to arrange a separate individual private, remote section of the AWS cloud on which you can launch AWS resources in a virtual network. Amazon VPC boosts your network framework to the cloud, making it easy to remain running essential enterprise applications after an on premise interruption. Amazon RDS is a cloud based relational database service that lets you run your management databases in the cloud after a disaster. You can also clone your database functioning in different territory. Azure's disaster recovery options can be 
Vol. 8, Issue 5, May 2019

refined into two significant services Azure storage and Azure site recovery. Azure storage is identical to S3 in that it supports cloud based object storage for your valuable data. Azure hand over its storage service as a managed service so sustenance and other concerns are managed for you. You can get your data from anyplace online via HTTP or HTTPS. Third party services can also fuse or incorporate with Azure to minimize Azure storage rate. The decreased costs come from executing non-standard processes to enhance storage efficiency, such as data deduplication and compression. Cloud providers generally compress stored data, but any cost accumulation from this are not ordinarily passed on to end users. Azure site recovery is a committed disaster recovery service that guarantees to lower application downtime for interruptions that arise and alter either on premise apps or on cloud based virtual apps. You can operate site recovery as a secondary environment for functioning of apps. You also have the choice of decreasing dependence on enterprise foundation generally by running your app mainly in the Azure cloud and utilizing site recovery for disaster recovery between various Azure zones. The service guarantees short recovery time objective (RTO) and recovery point objective (RPO), both of which are crucial objective in any disaster recovery plan. The RTO is the final mark time you set for recovering IT infrastructure that holds significant business exercises, while RPO is your company's tolerable level of data loss expressed in time. If a given entity has a 40-minute RPO, you need to back up that entity every 40 minutes.

\section{CONCLUSION}

The game of data got changed by the providers of cloud by providing secondary environments which are cost efficient. The two AWS and Azure yields disaster recovery options with excellent durability, scalability, and pay-as-you-go pricing options. AWS doesn't provide a committed loyal disaster recovery service you can use its services suite to address different aspects of disaster recovery, such as network, compute or storage. The benefit of AWS in this regard is that it offers more flexibility in choices, however, arguably provide everything you need for a successful disaster recovery strategy that utilizes the cloud and creates a large difference to the survivability of a business.

\section{REFERENCES}

[1]. Swedha K and Tanuja Dubey, “Analysis of Web Authentication methods using Amazon Web Services”, $9^{\text {th }}$ ICCCNT 2018, $10-12$ July 2018.

[2]. Amit S. Rodge, Chandan Pramanik, Joy Bose and Sandeep Kumar Soni, "Multicast Routing with Load Balancing Using Amazon Web Service", 2014 annual IEEE India Conference (INDICON).

[3]. Yorisan P. Baginda, Achmad Affandi and Istas Pratomo, "Analysis of RTO and RPO of a Service Stored on Amazon Web Service (AWS) and Google Cloud Engine (GCE)", $10^{\text {th }}$ International Conference on Information Technology and Electrical Engineering (ICITEE) 2018.

[4]. Patricia Takako Endo, "Minimizing and Managing Cloud Failures", IEEE Computer Society, November 2017.

[5]. P. Subhashini and Sindhura Nalla, "Data Retrieval Mechanism using Amazon Simple Storage Service and Windows Azure", 2016 International Conference on Computing for Sustainable Global Development (INDIACom).

[6]. Saakshi Narula, Arushi Jain and Ms. Prachi, "Cloud Computing Security", 2015 Fifth International Conference on Advanced Computing and Communication Technologies.

[7]. Robert Gyorodi, Marius Iulian Pavel, Cornelia Gyorodi and Doina Zmaranda, "Performance of OnPrem versus Azure SQL Server: A Case Study", IEEE Access 2019. 